\title{
Free-Breathing Whole-Heart Coronary MRA: Motion Compensation Integrated into 3D Cartesian Compressed Sensing Reconstruction
}

\author{
Christoph Forman ${ }^{1,2}$, Robert Grimm ${ }^{1}$, Jana Maria Hutter ${ }^{1,2}$, \\ Andreas Maier ${ }^{1,2}$, Joachim Hornegger ${ }^{1,2}$, and Michael O. Zenge ${ }^{3}$ \\ 1 Pattern Recognition Lab, Department of Computer Science, Friedrich-Alexander \\ Universität Erlangen-Nürnberg, Erlangen, Germany \\ 2 Erlangen Graduate School in Advanced Optical Technologies (SAOT), \\ Friedrich-Alexander Universität Erlangen-Nürnberg, Erlangen, Germany \\ 3 MR Application and Workflow Department, Healthcare Sector, Siemens AG, \\ Erlangen, Germany
}

\begin{abstract}
Respiratory motion remains a major challenge for wholeheart coronary magnetic resonance angiography (CMRA). Recently, iterative reconstruction has been augmented with non-rigid motion compensation to correct for the effects of respiratory motion. The major challenge of this approach is the estimation of dense deformation fields. In this work, the application of such a motion-compensated reconstruction is proposed for accelerated 3D Cartesian whole-heart CMRA. Without the need for extra calibration data or user interaction, the nonrigid deformations due to respiratory motion are directly estimated on the acquired image data. In-vivo experiments on 14 healthy volunteers were performed to compare the proposed method with the result of a navigator-gated reference scan. While reducing the acquisition time by one third, the reconstructed images resulted in equivalent vessel sharpness of $0.44 \pm 0.06 \mathrm{~mm}^{-1}$ and $0.45 \pm 0.05 \mathrm{~mm}^{-1}$, respectively.
\end{abstract}

\section{Introduction}

The absence of ionizing radiation renders whole-heart coronary magnetic resonance angiography (CMRA) as an appealing alternative to computed tomography angiography. However, uncompensated motion during the data acquisition remains a challenging problem in CMRA which is not yet fully solved. The effects of cardiac motion are sufficiently minimized by segmented acquisitions triggered with an electrocardiogram (ECG) to end-diastole. For each heart beat, only a restricted amount of data can be acquired during this resting phase of the heart. This prolongs the acquisition time and leaves whole-heart imaging susceptible to respiratory motion. The consequences are reconstruction artifacts, such as blurring or aliasing, which significantly impair an accurate depiction of the coronary arteries. Most commonly, respiratory motion is compensated prospectively by gating the acquisition with a navigator [1. However, acceptance rates less than $50 \%$ extend the scan time unfavorably. 
Therefore, retrospective motion correction approaches were proposed to overcome these disadvantages in scan time efficiency. With self-navigation [23], the position of the heart is detected in readouts acquired in the main direction of the respiratory motion of the heart, namely in the superior-inferior (SI) direction. Although this method provides full efficiency, the respiratory motion detection and correction is limited to a translation in one dimension. Recently, iterative reconstruction was augmented with image registration 445, which incorporates the compensation of non-rigid deformations during data acquisition. Assuming that the acquired data can be segmented into subsets with minimum motion, image registration can be used for the compensation of motion between the individual subsets [6]. Alternatively, the estimation of the motion model has been integrated into the image reconstruction [5 7] without the need for previously reconstructed images. Although promising results have been demonstrated, most of the MR applications utilizing such approaches were limited to $2 \mathrm{D}$ cine imaging [5 899. This is mainly due to the lack of sufficient data for estimating a dense deformation field. The first application to 3D whole-heart CMRA has been proposed by Schmidt et al. 10. However, this approach requires the acquisition of extra calibration data to generate the motion model. In the current work, a motion compensated reconstruction is presented for 3D Cartesian whole-heart CMRA that estimates the non-rigid deformations directly from the acquired image data without the need for extra acquisitions. The proposed approach is evaluated in in-vivo experiments on 14 volunteers and the resulting vessel sharpness is compared to a navigator-gated reference scan.

\section{Materials and Methods}

Free-breathing whole-heart CMRA is performed with data acquisition in segments of readouts distributed over multiple heart beats, henceforth denoted as a shot. The Cartesian variable-density spiral phyllotaxis pattern [1] is used for the sampling within the phase-encoding plane. This pattern provides smooth gradient waveforms for the trajectory of each shot to avoid eddy current effects in the balanced steady-state free-precession (bSSFP) data acquisition. Furthermore, the incoherent sampling pattern renders this trajectory well-suited for accelerated Cartesian imaging and compressed sensing reconstruction [12.

As illustrated in Fig. 1, the compensation of respiratory motion in image reconstruction 9 requires 1 ) the detection of respiratory motion in the acquired data, 2) the generation of a motion model, and finally 3) a reconstruction utilizing this model to estimate the motion-free volume.

\subsection{Respiratory Motion Detection}

In order to facilitate a minimum planning effort and the detection of respiratory motion, data is acquired in sagittal slab orientation to capture the main direction of respiration within each readout. An additional readout through k-space center is performed prior to the acquisition of each shot. The Fourier transform of this 


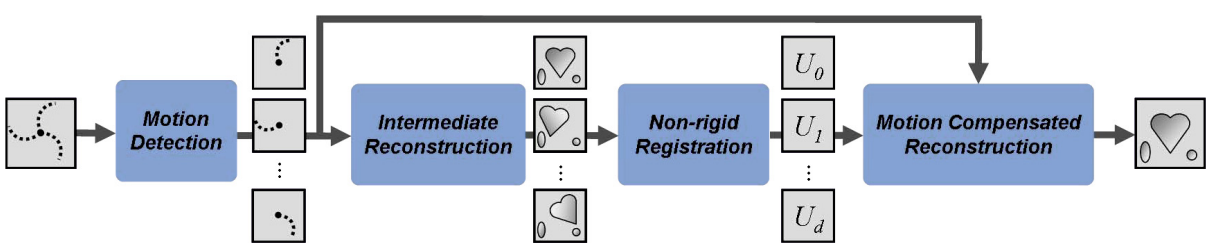

Fig. 1. Block diagram of the motion-compensated reconstruction: First, respiratory motion is detected and the acquired data are segmented into motion states. After an intermediate reconstruction of the individual motion states, a motion model is estimated using image registration. Finally, the motion-free volume is reconstructed incorporating the motion model.

readout provides an orthogonal projection of the imaging volume. In this projection, the effects of respiratory motion are detected as a discrete $1 \mathrm{D}$ translational offset relative to a reference using the self-navigation principle [3. Subsequently acquired data of each shot is assigned to the detected offset. Uncorrected respiratory motion during data scan leads to inconsistencies in the acquired data, which results in motion artifacts in the reconstructed volumes. Hence, a binning of the data according to the detected offsets into a set of finite motion states denoted by $D$ allows to minimize residual motion within each bin.

\subsection{Generation of the Motion Model}

In the next step, a motion model is generated using image registration and an intermediate reconstruction of the acquired image data, which has been segmented in respiratory motion states.

Intermediate Reconstruction. First, each volume $\tilde{\mathbf{x}}_{d}$ assigned to the detected offsets $d \in D$ is reconstructed. However, since the acquired data is already undersampled, the sampling rate in each respiratory motion state is further decreased by the binning process. As a consequence, none of the individual motion states contains sufficient data to satisfy the Nyquist criterion. But, compressed sensing allows the reconstruction of such undersampled data, while minimizing incoherent artifacts by enforcing a sparse representation. Thereby, registration errors can be avoided in the estimation of the motion model on basis of the intermediately reconstructed volumes. In order to further support the reconstruction of each volume $\tilde{\mathbf{x}}_{d}$, readouts from adjacent motion states are taken into account. Because the effect of motion on the acquired data increases with the respiratory offset relative to $d$, the readouts are weighted according to this distance using a scaled Gaussian kernel where the maximum is 1 . While $\mu$ is set to the detected offset of each bin $d$, the impact of motion on the reconstructed images is adjusted by $\sigma$. Although acquisitions from adjacent bins are considered, some motion states have insufficient data for image reconstruction. Thus, only respiratory states $D_{0.05}$ with an sampling rate greater than 0.05 compared to 
the fully sampled k-space are used for further processing. A diagonal matrix $\mathbf{W}_{d}$, containing the estimated weights for each $\mathrm{k}$-space sample and respiratory phase, is directly introduced into the data fidelity term of the cost function of the iterative reconstruction:

$$
f\left(\tilde{\mathbf{x}}_{d}\right)=\underset{\tilde{\mathbf{x}}_{d}}{\operatorname{argmin}}\left\|\mathbf{W}_{d}\left(\mathbf{A} \tilde{\mathbf{x}}_{d}-\mathbf{y}\right)\right\|_{2}^{2}+\lambda\left\|\tilde{\mathbf{x}}_{d}\right\|_{\mathrm{TV}} \quad \forall d \in D_{0.05}
$$

where the first part of the cost function optimizes the data fidelity of the reconstructed volume, written as a concatenated vector, $\tilde{\mathbf{x}}_{d} \in \mathbb{C}^{n_{x} n_{y} n_{z}}$ with respect to the measured data $\mathbf{y} \in \mathbb{C}^{n_{k} n_{c}}$ with $n_{k}$ acquired k-space samples from $n_{c}$ coil elements. A represents the MR system matrix including the receiver coil sensitivities, the Fourier transform, and the sampling pattern. In the second term, artifacts due to the incoherent sampling are penalized during image reconstruction by enforcing a sparse representation of the estimated volume in the total variation (TV) norm [13]. The described objective function is solved using the limited-memory Broyden-Fletcher-Goldfarb-Shanno (L-BFGS) method [14.

Non-rigid Registration. Finally, the reconstructed volumes are used as input for image registration to estimate the motion model. The compensation of nonrigid deformations in the motion-compensated reconstruction requires a dense pixel-wise deformation field and its inverse for every respiratory motion state. Here, both forward and backward deformations are estimated using non-rigid 3D-3D symmetric diffeomorphic registration [15. The volume reconstructed from the end-expiratory motion state, which contains the most acquisitions, serves as a reference and is consecutively registered to all other volumes.

\subsection{Motion Compensated Reconstruction}

After this, the estimated deformation fields are used to generate the motion matrices $\mathbf{U}_{d}$ of size $n_{x} n_{y} n_{z} \times n_{x} n_{y} n_{z}$, which are incorporated into the data fidelity term for the reconstruction of the motion-free volume $\tilde{\mathbf{x}}$ :

$$
f(\mathbf{x})=\underset{\mathbf{x}}{\operatorname{argmin}} \sum_{d \in D_{0.05}}\left\|\mathbf{A} \mathbf{U}_{d} \mathbf{x}-\mathbf{y}_{d}\right\|_{2}^{2}+\lambda\|\mathbf{x}\|_{\mathrm{TV}},
$$

where $\mathbf{U}_{d}$ deforms $\mathbf{x}$ into the $d$ th motion state to facilitate a comparison to the acquired data in this respiratory phase.

\subsection{MR Experiments}

In-vivo experiments were performed in 14 healthy volunteers (male, age 24.6 \pm 5.3 ) on a $1.5 \mathrm{~T}$ clinical MR scanner (MAGNETOM Aera, Siemens AG, Healthcare Sector, Erlangen, Germany), with software release syngo MR D13A. Signal reception was performed using an 18-channel body matrix coil and 8 elements of a spine matrix coil. All measurements were ECG-triggered. The spiral phyllotaxis sampling pattern was generated for the proposed method with 377 shots, and 
for the navigator-gated reference scan with 233 shots, containing 30 readouts each. Compared to a fully sampled k-space, this results in a sampling ratio of 0.246 and 0.152 , respectively. Data acquisition was performed during the end diastolic phase of the cardiac cycle. At the beginning of each shot, an additional readout for the detection of respiratory motion was acquired. The individual trigger delay to the subject specific cardiac resting period was adjusted using a two-dimensional cine scan with axial slice orientation performed prior to the coronary acquisition. The whole-heart imaging volume was placed in sagittal slab orientation with the blood pool of the heart positioned in the center of the FOV. 3D volume-selective, T2-prepared, fat-saturated bSSFP imaging was performed with the following parameters: TR/TE 4.0/2.0 ms, radio frequency $(\mathrm{RF})$ excitation angle $115^{\circ}$, FOV $270 \times 270 \times 167 \mathrm{~mm}^{3}$, acquired matrix $256 \times 218 \times 150$, reconstructed matrix $256 \times 256 \times 176$, slice oversampling $22 \%$, voxel size $1.05 \mathrm{~mm}^{3}$ and a receiver bandwidth of $849 \mathrm{~Hz} / \mathrm{Px}$. For the navigator-gated acquisition an acceptance window with a width of $5 \mathrm{~mm}$ was placed in end-expiration.

The acquired data of the proposed method was reconstructed with three different methods: $\mathrm{M}_{1}$ ) using Eq. 1 with $\mathbf{W}$ being the identity matrix, $\mathrm{M}_{2}$ ) with $\mathbf{W}$ weighting the input data towards end-expiration with $\sigma=1.25$, and $\mathrm{M}_{3}$ ) with motion compensation following Eq. 2. The result of all methods were compared to the reconstruction of an additional navigator-gated reference scan $\left(\mathrm{M}_{\mathrm{Ref}}\right)$. In all cases, the iterative reconstruction was performed with $\lambda=0.00002$ and was terminated after a fixed number of 8 iterations, where no considerable improvement of the fidelity was observed.

\subsection{Data Analysis}

For the assessment of the image quality, vessel sharpness was compared in the resulting volumes obtained from all methods. A centerline was manually segmented for the right coronary artery (RCA) in the 3D isotropic volumes using CoronaViz (Work-in-Progress software, Siemens Corporate Research, Princeton, NJ, USA). Vessel sharpness was evaluated in the proximal and distal part of the RCA. For the proximal evaluation, five points were selected in the volumes obtained from all methods at corresponding positions distributed over the first $40 \mathrm{~mm}$ of each centerline. Accordingly, three points were selected in the subsequent $25 \mathrm{~mm}$ of the vessel for the distal evaluation. At each measurement point, a cross-section was sampled perpendicular to the centerline. On this cross-section the sharpness was evaluated by the inverse of the average distance between $20 \%$ and $80 \%$ of the maximum signal intensity on both sides as described in [16. Finally, vessel sharpness of each part of the coronary vessel was estimated by averaging the results of all cross-sections.

\section{Results and Discussion}

Whole-heart free-breathing acquisitions utilizing the self-navigation principle as well as those with navigator gating were performed successfully in all volunteers. 

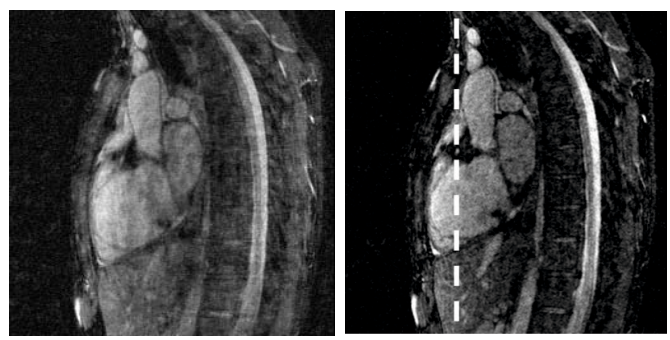

(a)

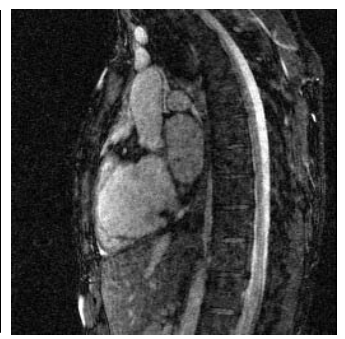

(b)

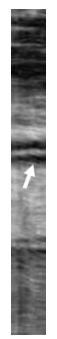

(c)

Fig. 2. (a) Sagittal slice of an intermediate reconstruction at end-inspiration, midrange, and end-expiration. A temporal profile is plotted along the dashed line over all respiratory states (b) pre and (c) post non-rigid motion compensation. The arrows in these profiles indicate the position of the right coronary artery.

The average navigator acceptance rate was $39.5 \pm 10.1 \%$, which prolonged the acquisition time to $10.1 \pm 2.3 \mathrm{~min}$. Although the sampling pattern for the proposed method was generated for $60 \%$ more shots compared to the reference scan, the acquisition time was reduced to $6.3 \pm 0.9 \mathrm{~min}$. This means a reduction of the proposed method by $37 \%$.

Table 1 shows the qualitative results of vessel sharpness for all methods and volunteers. On average, in $\mathrm{M}_{1}$ the effects of motion lead to a vessel sharpness of $0.381 \pm 0.047 \mathrm{~mm}^{-1}$ (proximal) and $0.362 \pm 0.039 \mathrm{~mm}^{-1}$ (distal). With $\mathrm{M}_{2}$ the sharpness is improved to $0.426 \pm 0.057 \mathrm{~mm}^{-1}$ (proximal) and $0.427 \pm 0.043 \mathrm{~mm}^{-1}$ (distal) and with $\mathrm{M}_{3}$ to $0.444 \pm 0.056 \mathrm{~mm}^{-1}$ (proximal) and $0.441 \pm 0.046 \mathrm{~mm}^{-1}$ (distal). A subsequent reference scan $\mathrm{M}_{\text {Ref }}$ resulted in an equivalent image quality with $0.449 \pm 0.051 \mathrm{~mm}^{-1}$ (proximal) and $0.447 \pm 0.050 \mathrm{~mm}^{-1}$ (distal).

The respiratory motion detection resulted in 8 to 18 motion states. Fig. 2(a) shows a sagittal slice of the reconstructed intermediate volume weighted to endinspiration, mid-range, and end-expiration of volunteer 5. In this image, a temporal profile along the dashed line over all intermediate volumes is plotted in Fig. 2(b). The arrow indicates the impact of respiration on the right coronary artery. As seen in Fig. 2(c) the effects of respiratory motion were minimized after application of the inverse deformation from the estimated motion model. For this volunteer, Fig. 3 shows the reformatted images of the coronary arteries obtained from all methods. The $\mathrm{M}_{1}$ reconstruction exhibits motion artifacts, which affects the proximal part of the coronary vessel right after extension from the aorta as indicated by the top arrow in Fig. 3(a). In this region, the RCA can be recovered by weighting the reconstruction to end-expiration in $\mathrm{M}_{2}$ as shown in Fig. 3(b). However, this weighting introduces an additional sub-sampling on the acquired data. The reduced available data for reconstruction induced a loss of the overall sharpness in the image. Thereby, the coronary vessel is hardly visible in the distal part of the heart as denoted by the bottom arrow. Especially, the vessel sharpness in this subsection of the coronary benefits from incorporating all data in image reconstruction as performed in $\mathrm{M}_{3}$, Fig. $3(\mathrm{c})$. While reducing the 


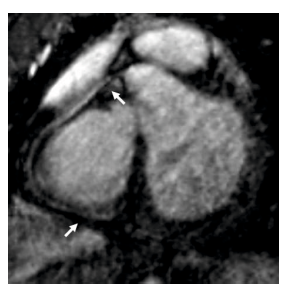

(a)

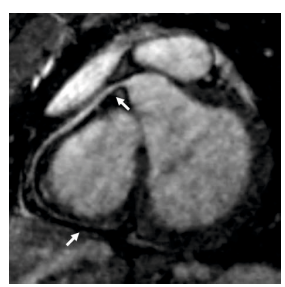

(b)

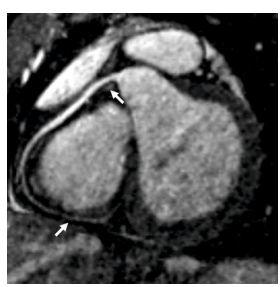

(c)

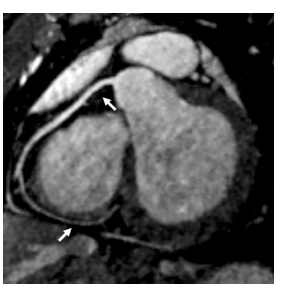

(d)

Fig. 3. The reformatted images of the RCA of volunteer 5 reconstructed with (a) no motion correction, (b) weighted reconstruction with $\mu$ set to end-expiration, and (c) motion-compensated reconstruction incorporating the motion model. (d) An additional scan with navigator gating is performed for reference.

Table 1. Numerical results for the comparison between the reconstruction of the data without motion compensation $\left(\mathrm{M}_{1}\right)$, weighted to end-expiration $\left(\mathrm{M}_{2}\right)$, incorporating the motion model $\left(\mathrm{M}_{3}\right)$, and of the additional scan with navigator gating $\left(\mathrm{M}_{\mathrm{Ref}}\right)$. In cases denoted by "-" the coronary artery was not fully detectable in the distal part.

\begin{tabular}{|c|c|c|c|c|c|c|c|c|c|c|c|c|c|c|}
\hline & 1 & 2 & 3 & 4 & 5 & 6 & 7 & 8 & 9 & 10 & 11 & 12 & 13 & 14 \\
\hline $\mathbf{M}_{1}$ & 0.366 & 0.394 & 0.354 & 0.421 & 0.401 & 0.349 & 0.313 & 0.390 & 0.513 & 0.396 & 0.374 & 0.351 & 0.344 & 0.363 \\
\hline $\mathbf{M}_{2}$ & 0.495 & 0.452 & 0.358 & 0.470 & 0.463 & 0.367 & 0.367 & 0.434 & 0.560 & 0.407 & 0.408 & 0.385 & 0.399 & 0.404 \\
\hline $\mathbf{M}_{3}$ & 0.511 & 0.474 & 0.429 & 0.475 & 0.500 & 0.383 & 0.369 & 0.458 & 0.568 & 0.426 & 0.420 & 0.405 & 0.395 & 0.402 \\
\hline$M_{\text {Ref }}$ & 0.551 & 0.480 & 0.449 & 0.482 & 0.500 & 0.376 & 0.396 & 0.492 & 0.490 & 0.439 & 0.423 & 0.391 & 0.418 & 0.403 \\
\hline $\mathbf{M}_{1}$ & 0.371 & 0.394 & - & 0.422 & - & 0.333 & - & 0.386 & 0.376 & 0.350 & 0.383 & 0.308 & 0.299 & - \\
\hline $\mathbf{M}_{2}$ & 0.434 & 0.442 & - & 0.508 & 0.438 & 0.386 & - & 0.474 & 0.465 & 0.354 & 0.426 & 0.419 & 0.392 & 0.387 \\
\hline $\mathbf{M}_{3}$ & 0.432 & 0.468 & - & 0.509 & 0.472 & 0.383 & - & 0.481 & 0.494 & 0.362 & 0.425 & 0.454 & 0.406 & 0.408 \\
\hline$M_{\text {Ref }}$ & 0.489 & 0.470 & 0.377 & 0.509 & 0.478 & 0.391 & 0.350 & 0.482 & 0.506 & 0.410 & 0.451 & 0.451 & 0.480 & 0.413 \\
\hline
\end{tabular}

acquisition time, the vessel sharpness of this image showed comparable results as $\mathrm{M}_{\text {Ref }}$ illustrated in Fig. $3(\mathrm{~d})$.

The presented work uniquely demonstrates the estimation of non-rigid displacement fields for motion-compensated reconstruction directly from the image data. Compared to a navigator-gated reference, the proposed approach resulted in an equivalent vessel sharpness, while reducing the acquisition time by one third. Furthermore, the described approach not only renders additional calibration data unnecessary, but also does not require further user interaction. This improvement to the workflow can hardly be overestimated, since CMRA has not been widely used in clinical practice due to the complexity of the examination. Therefore, a high priority should be given to the integration of the proposed method into the clinical routine.

Disclaimer: The concepts and information presented in this paper are based on research and are not commercially available.

Acknowledgements. The authors gratefully acknowledge funding of the Erlangen Graduate School in Advanced Optical Technologies (SAOT) by the German Research Foundation (DFG) in the framework of the German excellence initiative. 


\section{References}

1. Stuber, M., Botnar, R.M., Danias, P.G., Kissinger, K.V., Manning, W.J.: Submillimeter Three-dimensional Coronary MR Angiography with Real-time Navigator Correction: Comparison of Navigator Locations. Radiology 212, 579-587 (1999)

2. Stehning, C., Börnert, P., Nehrke, K., Eggers, H., Stuber, M.: Free-Breathing Whole-Heart Coronary MRA With 3D Radial SSFP and Self-Navigated Image Reconstruction. Magn. Reson Med. 54, 476-480 (2005)

3. Piccini, D., Littmann, A., Nielles-Vallespin, S., Zenge, M.O.: Respiratory SelfNavigation for Whole-Heart Bright-Blood Coronary MRI: Methods for Robust Isolation and Automatic Segmentation of the Blood Pool. Magn. Reson Med. 68, 571-579 (2012)

4. Batchelor, P.G., Atkinson, D., Irarrazaval, P., Hill, D.L., Hajnal, J., Larkman, D.: Matrix Description of General Motion Correction Applied to Multishot Images. Magn. Reson Med. 54, 1273-1280 (2005)

5. Odille, F., Vuissoz, P.A., Marie, P.Y., Felbinger, J.: Generalized Reconstruction by Inversion of Coupled Systems (GRICS) Applied to Free-Breathing MRI. Magn. Reson Med. 60, 146-157 (2008)

6. Boldea, V., Sharp, G.C., Jiang, S.B., Sarrut, D.: 4D-CT lung motion estimation with deformable registration: Quantification of motion nonlinearity and hysteresis. Med. Phys. 35(3), 1008-1018 (2008)

7. Hinkle, J., Szegedi, M., Wang, B., Salter, B., Joshi, S.: 4D CT image reconstruction with diffeomorphic motion model. Med. Imag. Anal. 16, 1307-1316 (2012)

8. Xue, H., Ding, Y., Guetter, C., Jolly, M.-P., Guehring, J., Zuehlsdorff, S., Simonetti, O.P.: Motion compensated magnetic resonance reconstruction using inverseconsistent deformable registration: Application to real-time cine imaging. In: Fichtinger, G., Martel, A., Peters, T. (eds.) MICCAI 2011, Part I. LNCS, vol. 6891, pp. 564-572. Springer, Heidelberg (2011)

9. Usman, M., Atkinson, D., Odille, F., Kolbitsch, C., Vaillant, G., Schaeffter, T., Batchelor, P.G., Prieto, C.: Motion Corrected Compressed Sensing for FreeBreathing Dynamic Cardiac MRI. Magn. Reson Med. (online)

10. Schmidt, J.F.M., Buehrer, M., Boesiger, P., Kozerke, S.: Nonrigid Retrospective Respiratory Motion Correction in Whole-Heart Coronary MRA. Magn. Reson Med. 66, 1541-1549 (2011)

11. Forman, C., Piccini, D., Hutter, J., Grimm, R., Hornegger, J., Zenge, M.O.: Accelerated Whole-Heart Coronary Imaging using Multiple Breath-holds and Compressed Sensing Monitored by Self-Navigation. In: Proc. ISMRM 2012, p. 1157 (2012)

12. Lustig, M., Donoho, D., Pauly, J.M.: Sparse MRI: The Application of Compressed Sensing for Rapid MR Imaging. Magn. Reson Med. 58, 1182-1195 (2007)

13. Rudin, L.I., Osher, S., Fatemi, E.: Nonlinear total variation based noise removal algorithms. Physica D 60, 259-268 (1992)

14. Nocedal, J.: Updating Quasi-Newton Matrices with Limited Storage. Math. Comp. 35, 773-782 (1980)

15. Avants, B.B., Epstein, C.L., Grossman, M., Gee, J.C.: Symmetric diffeomorphic image registration with cross-correlation: Evaluating automated labeling of elderly and neurodegenerative brain. Med. Imag. Anal. 12, 26-41 (2008)

16. Li, D., Carr, J.C., Shea, S.M., Zheng, J., Deshpande, V., Wielopolski, P.A., Finn, J.P.: Coronary Arteries: Magnetization-prepared Contrast-enhanced Threedimensional Volume-targeted Breath-hold MR Angiography. Radiology 219, 270-277 (2001) 